Interference Control in Working Memory

Krishneil A. Singh ${ }^{1}$, Gilles E. Gignac ${ }^{1}$, Christopher R. Brydges ${ }^{1}$, Alodie Rey-Mermet ${ }^{2}, \&$ Ullrich K. H. Ecker ${ }^{1}$

\author{
${ }^{1}$ School of Psychological Science, University of Western Australia \\ ${ }^{2}$ General Psychology, Catholic University of Eichstätt-Ingolstadt
}

Word Count: 8,221 (excluding title page, abstract, references, tables, and figure captions)

Address for Correspondence:

Kris Singh

School of Psychological Science

University of Western Australia

35 Stirling Highway, Perth, WA 6009, Australia

Email: kris.singh@ research.uwa.edu.au 


\begin{abstract}
Working memory (WM) is a limited-capacity system requiring an interference-control process to avoid being cluttered from irrelevant information. Recently, it has been suggested that this "housekeeping" mechanism can be attributed to an item-wise removal process serving to actively remove irrelevant information from WM. It has been theorized that this active removal process serves to facilitate both WM maintenance in the face of distraction as well as the updating of outdated information. An alternate view, however, is that interference control in WM relies on an inhibitory process that suppresses the activation of distractors against competing, task-relevant representations. This study is the first to assess the extent to which removal and inhibition represent the same cognitive process. One-hundred and thirty-eight undergraduate students from the University of Western Australia $(M=20.42, S D=3.09)$ completed a novel removal task battery in addition to an inhibition task battery. Data were analysed using a hybrid path analytic and structural equation model. The modelling found that there was unique variance associated with the removal latent variable, and estimated that only approximately $9 \%$ of the variance in the removal latent variable could be accounted for by the inhibition tasks, providing tentative support that removal should not be considered a process of cognitive inhibition. The findings support previous claims that removal is an independent WM updating process. The findings are also consistent with a class of computational WM models proposing that removal and inhibition operate on different levels.
\end{abstract}




\section{Interference Control in Working Memory}

Working memory (WM) is a limited-capacity system that provides a workplace for ongoing cognition (Miyake \& Shah, 1999; Oberauer, 2009). To meet processing demands, WM is required to build representations that are both stable and flexible. On the one hand, maintenance processes provide the stability to protect relevant WM representations from forgetting and interference from outdated or irrelevant content. On the other hand, updating processes provide the flexibility to manipulate or replace outdated subsets of information in WM to reflect changes in informational relevance (e.g., when there are changes in the environment). Thus, WM maintenance and WM updating both involve resolving conflict between competing relevant and irrelevant memory representations.

The ability to resolve such conflict in a limited-capacity system has been proposed as an important individual-differences variable. For example, individual differences in interference control have been shown to predict WM capacity (e.g., Engle \& Kane, 2004; Engle, Kane, \& Tuholski, 1999) and fluid intelligence (e.g., Unsworth, 2010). However, there is debate surrounding the underlying cognitive processes responsible for achieving interference control (Ecker, Lewandowsky, \& Oberauer, 2014; Ecker, Oberauer, \& Lewandowsky, 2014; Fawcett \& Taylor, 2008, 2010, 2012; Festini \& Reuter-Lorenz, 2017; Hasher, Lustig, \& Zacks, 2007; Kane \& Engle, 2003). Recently, Ecker and colleagues (Chang, Ecker, \& Page, 2017; Ecker, Lewandowsky et al., 2014; Ecker, Oberauer et al., 2014; Oberauer, Lewandowsky, Farrell, Jarrold, \& Greaves, 2012; Oberauer \& Lewandowsky, 2016) proposed that there is an active, item-wise removal process that facilitates both maintenance and updating of WM. With respect to maintenance, removing irrelevant information serves to minimize interference and effectively increases WM capacity (Oberauer et al., 2012; Oberauer \& Lewandowsky, 2016). With respect 
to updating, efficiently removing outdated information facilitates the substitution of information in WM (Chang et al., 2017; Ecker, Lewandowsky, Oberauer, \& Chee, 2010; Ecker, Lewandowsky et al., 2014; Ecker, Oberauer et al., 2014).

Ecker et al. (Ecker, Lewandowsky et al., 2014; Ecker, Oberauer et al., 2014) obtained evidence for a removal process by modifying an updating task used by Kessler and Meiran (2008). This task measured the time it took people to replace items held in WM. More specifically, during each trial, participants encoded a set of items and individual items were then repeatedly replaced with newer items before a final recall of the set was required. Each updating step required a key press to indicate that participants had successfully updated their WM. The reaction time (RT) from the key press was used as a proxy for updating efficiency. However, because participants did not know what item was going to be replaced, Ecker et al. argued that the RT measure obtained for each update confounded the core updating process of removal with the generic operations of attention reorienting and encoding, because all three processes could only begin when the new item was presented.

In order to disentangle these processes, Ecker et al. (Ecker, Lewandowsky et al., 2014; Ecker, Oberauer et al., 2014) modified the updating phase of the task. In the new task, the to-beupdated item was cued to let participants know which item was going to be updated (i.e., the tobe-updated item's frame turned bold and red) before a new replacement item was presented. Additionally, the time between cue onset and presentation of the new item (i.e., the cue-target interval, CTI) was varied. Ecker et al. argued that people could use the CTI to remove irrelevant memory representations; thus, varying the CTI essentially meant varying the time permitted to remove an item from WM. It was hypothesised that a short CTI (e.g., $200 \mathrm{~ms}$ ) only allowed participants to focus attention on the to-be-updated frame. Therefore, reaction times in this 
condition, measured from onset of the new updated item, were expected to be longer because they included the time taken to remove the old, outdated item as well as the time taken to encode the new, updated item. By comparison, with a long CTI (e.g., 1,500 ms), the longer period between cue onset and the presentation of the new stimulus allowed participants to remove the cued item prior to the presentation of the new, updated item. Thus, RTs in this condition were expected to be shorter because they mainly included the time taken to encode the new item. As expected, updating latencies in the long-CTI condition were indeed significantly faster than in the short-CTI condition. The difference in updating latencies between the two CTI conditions supported Ecker et al.'s notion that people use the CTI to remove representations from WM.

Further evidence for an active, item-wise removal process comes from two phenomena: (1) an item-repetition effect, and (2) an item-similarity effect. The item-repetition effect in Ecker, Lewandowsky et al. (2014) demonstrated that if there was occasional repetition of items in the updating task, updating RTs were shorter when the updated item was the same as the outdated item; however, this repetition benefit was strongly reduced in the long-CTI condition. This indicates that item repetition can only be beneficial during an updating task to the degree that the outdated item is still in WM. This finding was thus taken as additional support for the removal notion. Similarly, a beneficial effect of item similarity was also found to depend on the time available for removal. Lendínez, Pelegrina, and Lechuga (2011) had reported an item-similarity effect in an updating task involving two-digit numbers. In this task, updating was quicker when the new updated item was numerically close to the outdated item (e.g., updating from 83 to 85 ) rather than numerically distant (e.g., updating from 83 to 97). Ecker, Lewandowsky et al. (2014) replicated this finding, while also manipulating the CTI. They found that the similaritydependent facilitation was more evident in the short-CTI condition than in the long-CTI 
condition. This indicates that updating benefitted from item similarity only to the extent that the outdated item had not been removed (i.e., in the short-CTI condition). Thus, Ecker, Lewandowsky et al. (2014) concluded that faster updating RTs associated with item repetition or item similarity are a result of representational overlap between outdated and new items. However, the more opportunity there is for an item representation to be removed before the updated item can be encoded, the less overlap — and thus the less facilitation from repetition or similarity—-there will be. These results support the view that an active removal process facilitates the updating of information in WM.

Ecker, Oberauer et al. (2014) additionally argued that the removal process may be important for general WM function to the extent that it serves to remove distracting information from WM and allows for more focussed processing of relevant information. Indeed, individuals with better removal efficiency have been shown to have a greater WM capacity (Singh, Ecker, \& Gignac, 2017). In this vein, the removal of irrelevant memory representations has been implemented into computational models of WM. In the SOB model by Farrell and Lewandowsky (2002), WM maintenance relies on bindings between item representations and spatiotemporal context markers. In the most recent version of this model, removal has been implemented as a Hebbian anti-learning process that unbinds item representations from their context markers (e.g., when remembering a series of digits such as $2-5-3$, unbinding the item "2" from its context marker "first position"). In other words, removal has been modelled as a process that undoes the association created between an irrelevant representation (e.g., a distractor item in a complex-span task, or an outdated item in an updating task) and its position marker during encoding, such that the distractor representation is gradually removed from working memory (Oberauer et al., 2012; Oberauer \& Lewandowsky, 2016). This implies that representations that are removed from WM 
are "unbound" but not entirely "deleted" and thus often remain recoverable. In the terminology of Oberauer (e.g., Oberauer, 2009), the item may be removed from the direct-access region of working memory, but may still remain accessible through its representation in activated longterm memory (LTM).

In summary, various authors (Chang et al., 2017; Ecker, Lewandowsky et al., 2014; Ecker, Oberauer et al., 2014; Oberauer et al., 2012; Oberauer \& Lewandowsky, 2016) have proposed that the notion of active, item-wise removal can be seen as a "new" type of executive function assisting with interference control in the service of both maintenance and updating of WM. An alternative, or complementary, position has been to characterize the processes underlying interference control in WM as inhibitory suppression processes. Specifically, it has been suggested that interference control relies on the suppression of a distracting representation amongst competing relevant representations. In other words, an outdated/irrelevant item's representation is weakened so that it becomes less accessible, and is therefore not selected against more relevant representations during retrieval (Dagry, Vergauwe, \& Barrouillet, 2017; Festini \& Reuter-Lorenz, 2017; Hasher et al., 2007; Hasher, Zacks, \& May, 1999; Healey, Campbell, Hasher, \& Ossher, 2010).

This position assumes that irrelevant information is actively suppressed, allowing for more efficient processing of goal-relevant representations (Hasher et al., 1999). Independent evidence for an active, inhibitory process involved in interference control in WM comes from Fawcett and Taylor (2008). In an item-method directed forgetting task, the authors showed that intentional forgetting of an item can slow down responses on a secondary processing task for up to 2,600 ms after a 'forget' cue. Specifically, during the study phase of the task, participants were presented with a list of individual words, each followed by a memory instruction to either 
remember or forget the word. Participants were also presented with an asterisk either after the presentation of the study word or the memory instruction. The asterisk acted as a detection probe and was presented at variable time intervals (i.e., 1,400, 1,800, or 2,600 ms). After the study phase, participants were presented with a yes-no recognition task where their responses indicated whether they had previously seen the word during the study phase, regardless of the instruction to remember or forget. In addition to finding a directed-forgetting effect (i.e., better memory for to-be-remembered, TBR, compared to to-be-forgotten, TBF, words), Fawcett and Taylor found that post-instruction RTs were longer for TBF probes compared to TBR probes at shorter time intervals (i.e., 1,400 and 1,800 ms), but not at the longer time interval (i.e.. 2,600 ms). They interpreted this finding as evidence that intentionally forgetting items is just as cognitively demanding as remembering them, and attributed their findings to an active inhibition mechanism that limits processing of irrelevant, TBF words (Fawcett \& Taylor, 2008; see also Fawcett \& Taylor, 2010, 2012).

Additionally, Festini and Reuter-Lorenz (2017) presented evidence for an active control mechanism in WM. These authors assessed the accessibility of distractors also using an itemrecognition directed forgetting paradigm. They found that memory for TBF items from lists without competing TBR items was at least as poor as memory for TBF items from lists requiring concurrent retention of competing TBR items. This finding goes against the idea that the active maintenance of TBR items passively "pushes out" TBF items from WM and, in fact, supports the idea that an active inhibitory process, such as item inhibition (Festini \& Reuter-Lorenz, 2017), is engaged after a forget cue.

The results of Fawcett and Taylor (2008) and Festini and Reuter-Lorenz (2017) suggest that an active inhibition process reduces accessibility of irrelevant/outdated information in WM. 
Their results converge with literature from various other groups proposing that interference control in WM involves the suppression of irrelevant memory representations (e.g., Anderson, 2001; Bjork, 1989; Conway \& Engle, 1994; Engle, 1996; Hasher et al., 1999; Hasher et al., 2007; Healey et al., 2010; Zacks et al., 1996). The question arises whether these studies and the studies by Ecker and colleagues all investigated the same process, just with different interpretations; in other words, it is unclear whether Ecker et al.'s proposed removal process is in fact an inhibitory process.

Inhibition, as a broad construct, refers to stopping or overriding a cognitive process or behavioural response, in whole or in part (MacLeod, 2007). This definition suggests different forms of inhibition, and indeed, the literature proposes that inhibitory processes are a group of inter-related functions suggesting that inhibition is a non-unitary construct (e.g., Friedman \& Miyake, 2004; Harnishfeger, 1995; Hasher et al., 2007: Nigg, 2000). There have been several attempts to classify the major types of inhibitory processes into cohesive taxonomies (e.g., Harnishfeger, 1995; Hasher et al., 1999; Nigg, 2000).

Generally, these taxonomies have distinguished between cognitive and behavioural forms of inhibition, such that the suppression of mental processes is acknowledged to occur at an earlier stage than the suppression of impulses and motor responses (e.g., Bissett, Nee, \& Jonides, 2009; Nigg, 2000). On the behavioural level, Friedman and Miyake (2004) identified the ability to deliberately suppress dominant, automatic, or prepotent responses as a main inhibitory function; they labelled this process prepotent response inhibition (herein referred to as response inhibition, RI). Tasks measuring RI include the antisaccade task (Hallett, 1978), the stop-signal task (Logan, 1994), and the Stroop task (Stroop, 1935). Each of these tasks requires participants to withhold or suppress a dominant response. For example, in the antisaccade task, participants 
are required to suppress a reflexive saccade towards a cue on one side of the screen and instead look in the opposite direction to identify a target.

On the cognitive level of the inhibition taxonomy, Friedman and Miyake (2004) distinguished between two inhibitory processes that they labelled resistance to proactive interference (RPI) and resistance to distractor interference (RDI). Specifically, RPI refers to the ability to resist memory intrusions from previously relevant information that has since become irrelevant. Tasks measuring RPI include the Brown-Peterson paradigm (Kane \& Engle, 2000), the AB-AC-AD task (Rosen \& Engle, 1998), and the cued-recall task of Tolan and Tehan (1999). Each of these tasks present interfering information prior to the presentation of target information. For example, in the $\mathrm{AB}-\mathrm{AC}-\mathrm{AD}$ task, participants first learn a list of cue-target word pairs before learning a new list of targets that are paired with the same cues. The tasks, therefore, require participants to suppress previously relevant information.

By comparison, RDI refers to the ability to resist or resolve interference from distracting information that is irrelevant to the task at hand (Friedman \& Miyake, 2004). Tasks measuring RDI include the Eriksen flanker task (Eriksen \& Eriksen, 1974), the word naming task (Kane, Hasher, Stoltzfus, Zacks, \& Connelly, 1994), and the shape matching task (DeSchepper \& Treisman, 1996). Each of these tasks present interfering information simultaneously with target information. For example, in the Eriksen flanker task, participants are required to identify a target letter that is presented with letters flanking it, or identify the direction of a central arrow flanked by left- and right-pointing arrows. The task, therefore, requires participants to suppress the flankers so that they do not create interference with goal-relevant targets.

It can be argued that both RPI and RDI are conceptually similar to Ecker et al.'s (Ecker, Lewandowsky et al., 2014; Ecker, Oberauer et al., 2014) notion of removal. Regarding RPI, both 
item-specific removal and RPI are hypothesized to facilitate the updating of information in WM in that previously relevant representations are removed/suppressed before newer, more relevant representations are encoded into WM. Regarding RDI, both item-specific removal and RDI are thought to assist with the maintenance function of WM in that interference from irrelevant information is prevented via removal/inhibition to allow for focussed processing of task-relevant information.

Notably, however, Friedman and Miyake (2004) reported that RPI and RDI, arguably two similar inhibitory processes, were not significantly related $(r=-.03 ; p>.05 ; N=220)$. This finding was unexpected and went "against an intuitive notion that all types of resistance to interference are mediated by a common ability, regardless of the sources of the interference" (Friedman \& Miyake, 2004; p. 115). Oberauer (2009) suggested that the null relation found between RPI and RDI may be due to RPI tasks involving delayed recall of LTM associations that are more robust than the temporary bindings created in WM during tasks measuring RDI. Thus, while intuition suggests that different types of resistance to interference should relate with each other, the notion that RPI and RDI tasks may operate in different memory systems (LTM and WM, respectively) suggests one possible explanation for Friedman and Miyake's null relation. As discussed earlier, if removal can be characterized as an inhibitory process, then it should in theory relate to both RPI and RDI. However, given the ambiguity around the RPI tasks, the present study focussed on removal's potential relation to RDI.

The aim of this study was to provide an initial attempt to test the distinction between removal ability and inhibition ability in a large individual differences study using confirmatory and exploratory factor analytic methods. If removal is a process of cognitive inhibition, it would be expected that removal ability should relate to RDI; by contrast, it should be relatively 
dissimilar to RI, and should thus covary more with RDI than with RI. Alternatively, if removal is an independent non-inhibitory executive process, as proposed by Ecker et al. (Ecker, Lewandowky et al., 2014; Ecker, Oberauer et al., 2014), then there should be either no strong relation between removal and inhibition abilities, or removal should relate to both factors of inhibition equally (e.g., based on positive manifold; van der Maas, Dolan, Grasman, Wicherts, Huizenga, \& Raijmakers, 2006). Friedman and Miyake (2004) found that RDI and RI were related but separable constructs $(r=.67 ; p<.05 ; N=220)$. Both of these inhibitory processes have been frequently investigated in the literature. However, there is currently no empirical research that has investigated the extent to which removal and inhibition are related or reflect the same cognitive process.

\section{Method}

Participants. A total of 138 undergraduate students from the University of Western Australia participated for partial course credit. Based on various outlier criteria (see below), 16 participants were excluded from the analyses, yielding a final sample of $N=122$ participants (74 females, 48 males; age: $M=20.42$ years, $S D=3.09$; age range: 18 to 36 years).

\section{Materials.}

Updating tasks. Study 5 used the same three modified updating tasks that were used in Studies 1-4. Spearman-Brown corrected reliability indices, bootstrapped with $N=1,000$ runs, were $\rho=.46$ for the letter task, $\rho=.67$ for the digit task, and $\rho=.42$ for the word task. We noted that the reliabilities were lower than the reliabilities reported by Ecker, Lewandowsky et al. (2014). However, the present study used latent variables to represent the variables of interest. Consequently, as latent variables are devoid of measurement error (Fan, 2003), the reported 
effects were not considered affected by the relatively low reliabilities associated with the indicator observed scores.

Inhibition tasks. The inhibition task battery comprised six commonly used tests of inhibition. The battery was initially designed and implemented by Rey-Mermet, Gade, and Oberauer (in press) and modified for present purposes. These tests are assumed to tap into two forms of inhibition: (1) RI and (2) RDI. All tasks had accuracy and RT measures as dependent measures. All tasks included congruent and incongruent trials (4 of the 6 tasks also contained neutral trials; namely arrow flanker, local task, negative compatibility, and number Stroop; see details below). Participants completed a number of practice trials prior to the experimental trials. Feedback on performance was a happy/sad smiley face presented for $500 \mathrm{~ms}$ in the centre of the screen after every correct/incorrect trial, respectively. For each task, the dependent variable of interest was the RT difference between the incongruent and congruent trials (i.e., incongruentcongruent $)^{1}$. On average, as incongruent RTs were greater than congruent RTs, a positive difference was observed; greater (more positive) values reflect less efficient inhibition. The negative compatibility task was an exception: in this task, congruent trials produce longer RTs, which unlike the other tasks results on average in a negative RT difference; however, not only is the direction of the RT difference reversed, but also the interpretation of the effect: greater (more negative) values reflect more efficient inhibition. Thus, despite its reversed sign, the negativecompatibility measure was not recoded, to maintain consistency in terms of its covariation with other inhibition tasks as well as the removal task. The inhibition tasks are described briefly

\footnotetext{
${ }^{1}$ Congruence effects (i.e., RT difference scores between incongruent and congruent trials) were selected as the dependent variable following recommendations by Rey-Mermet, Gade, \& Oberauer, in press). Data were also analysed using regression residuals that predicted the incongruent RT from the congruent RT to control for processing speed, and it was found that the outcome was identical.
} 
below. Split-half Spearman-Brown corrected reliabilities for each of the inhibition tasks for both accuracy and RT measures are presented in Table 1.

\section{Response inhibition tasks.}

Antisaccade task (Hallet, 1978). The antisaccade task involved presentation of a visual cue and then a target stimulus. The cue (i.e., a black square) appeared either on the left or right side of the screen. Then, a target stimulus (i.e., a left-, upwards-, or right-pointing arrow) appeared in the cue position on either the left or right side of the screen. Participants were instructed to indicate the direction of the target arrow as quickly as possible. There were two conditions: (1) on prosaccade trials (i.e., congruent condition), the visual cue and target stimulus appeared on the same side of the screen; (2) on antisaccade trials (i.e., incongruent condition), the visual cue and the target stimulus appeared on opposite sides of the screen. The block structure was as follows: Each participant completed two blocks of prosaccade trials (50 trials per block; i.e., 100 experimental trials in total, plus 10 practice trials), and then two blocks of antisaccade trials (50 trials per block; i.e., 100 experimental trials in total, plus 24 practice trials).

During each trial, a fixation cross appeared in the centre of the screen for a variable amount of time (ranging from 1,500 to 3,250 ms in increments of $250 \mathrm{~ms}$ ). The cue was presented for $166 \mathrm{~ms}$. The initial target presentation time was $150 \mathrm{~ms}$ (as in Friedman \& Miyake, 2004), and target presentation time was varied using a weighted up-down method to avoid ceiling effects; that is, $17 \mathrm{~ms}$ were subtracted from the subsequent target presentation time when the response was correct, and $85 \mathrm{~ms}(5 \times 17 \mathrm{~ms})$ were added when the response was incorrect. The target stimulus was then masked by another image with arrows pointing left, upwards, and right for $300 \mathrm{~ms}$. A blank screen appeared until either a response was made or for a time that 
equalled $1,700 \mathrm{~ms}$ minus the target presentation time. Participants responded to the direction of the target arrow using the arrow keys with their index, ring, and middle finger.

Negative compatibility task (Briand, 1994). The negative compatibility task involved presentation of a visual prime and then a target stimulus. The prime (i.e., left- or right-pointing double arrow head, $\langle<$ or $>>$ ) appeared in the centre of the screen. Then, a target stimulus (i.e., also a left- or right-pointing double arrow head, $<<$ or $>>$ ) appeared either above or below the centre of the screen. Participants were instructed to indicate the direction of the target arrow head as quickly as possible. There were three conditions: (1) a congruent condition, where the target stimulus faced the same direction as the prime; (2) an incongruent (i.e., inhibition) condition, where the target stimulus faced the opposite direction as the prime; and (3) a neutral condition, where the prime consisted of equal signs (==). A mask, comprising both arrow head types and an equal sign superimposed on each other, was presented after the prime and before the target stimulus, in the centre of the screen. Each participant first completed one block of congruent trials ( 74 trials; plus 2 practice trials), followed by two blocks of incongruent trials ( 74 trials per block; i.e., 148 experimental trials in total, plus 4 practice trials), and another block of congruent trials (74 trials; plus 2 practice trials).

During each trial of the task, a fixation cross appeared in the centre of the screen for a variable amount of time (250, 350, or $500 \mathrm{~ms})$. Next, a blank screen was presented for $600 \mathrm{~ms}$ before the prime was presented for $30 \mathrm{~ms}$. The prime was masked for $100 \mathrm{~ms}$. The mask was followed by a blank screen for $50 \mathrm{~ms}$, before the target was presented for $100 \mathrm{~ms}$. Thus, the prime-target interval was $150 \mathrm{~ms}$, which is a delay that typically produces negative compatibility effects (Sumner, 2008). Following presentation of the target, a blank screen appeared until either 
a response was made or for a maximum response time of $1,900 \mathrm{~ms}$. Participants responded to the direction of the target arrow heads using the A and L keys for left and right, respectively.

Number Stroop task (Stroop, 1935). The number Stroop task involved the presentation of a target stimulus in the centre of the screen. The stimulus was a row of between 1 and 4 characters; characters were either 1's, 2's, 3's, 4's, or dollar symbols. Participants were instructed to indicate the number of characters in the row as quickly as possible, while suppressing reading the digits. There were three conditions: (1) a congruent condition, where the number of characters matched digit identity (e.g., 4444); (2) an incongruent condition, where the number of characters differed from digit identity (e.g., 222); and (3) a neutral condition, where dollar symbols were presented (e.g., \$\$). Each participant completed four blocks (74 trials per block; i.e., 296 experimental trials in total, plus 2 practice trials). During each trial of the task, a fixation cross appeared in the centre of the screen for $500 \mathrm{~ms}$. This was then followed by the presentation of the target stimulus, which appeared either until a response was made or for a maximum time of 2,000 ms. Participants responded using the number keys (i.e., 1, 2, 3, 4) in the upper number line of the keyboard.

\section{Resistance to distractor interference tasks.}

Arrow flanker task (Eriksen \& Eriksen, 1974). The arrow flanker task involved presentation of a target arrow placed centrally among four flanker arrows (two on each side) in the centre of the screen. Participants were instructed to indicate the direction of the target arrow as quickly as possible. There were three conditions: (1) a congruent condition, where the flanker arrows pointed in the same direction as the target arrow; (2) an incongruent condition, where flanker arrows pointed in the opposite direction as the target arrow; and (3) a neutral condition where only hyphens (i.e., -) flanked the target arrow. Each participant completed four blocks (74 
trials per block; i.e., 296 experimental trials in total, plus 2 practice trials). During each trial of the task, a fixation cross appeared in the centre of the screen for $500 \mathrm{~ms}$. After this, participants were presented with a row of five left- and right-pointing arrows (e.g., $\leftarrow, \rightarrow$ ) until either a response was made or for a maximum response time of 2,000 ms. Participants responded by pressing the A and L keys for left and right, respectively.

Local task (from the global-local task; Navon, 1977). The local component of the globallocal task involved presentation of a target stimulus, which was a large letter (i.e., Y, V, or Z) comprised of small letters (i.e., y's or v's), in the centre of the screen. Participants were instructed to identify the small letters as quickly as possible, while ignoring the large letter. There were three conditions: (1) a congruent condition, where the large letter consisted of the same small letters (e.g., a large V made up of small v's); (2) an incongruent condition, where the large letter consisted of different small letters (e.g., a large V made up of small y's); and (3) a neutral condition, where a large Z consisted of small v's or y's. Each participant completed two blocks (74 trials per block; i.e., 148 experimental trials in total, plus 2 practice trials). During each trial of the task, a fixation cross appeared in the centre of the screen for $500 \mathrm{~ms}$. After this, participants were presented with the target stimulus until either a response was made or for a maximum response time of 2,000 ms. Participants responded by pressing the A and L keys for $y$ and $\mathrm{v}$, respectively.

Simon task (Simon \& Ruddell, 1967). The Simon task involved the presentation of a target shape (i.e., a black square or circle), which appeared on either the left or right side of the screen. Participants were instructed to indicate whether the shape was a circle or a square as quickly as possible, while ignoring the position of the stimulus. There were two conditions: (1) a congruent condition, where the target was presented on the same side as the target's response 
key; and (2) an incongruent condition, where the target was presented on the opposite side to its response key. Each participant completed four blocks (50 trials per block; i.e., 200 experimental

trials, plus 2 practice trials). During each trial of the Simon task, a central fixation cross appeared in the centre of the screen for $500 \mathrm{~ms}$. After this, the target stimulus appeared on either the left or right side of the screen until either a response was made or for a maximum response time of 2,000 ms. Participants responded by pressing the A and L keys for square and circle, respectively.

Procedure. Participants first completed the three variants of the modified updating task (in the order of letters, digits, and words) before they completed the inhibition tasks. The inhibition task battery was administered in the following order: number Stroop, antisaccade, Simon, negative compatibility, local, and arrow flanker task. The entire experiment took approximately 90 minutes.

\section{Results}

Data screening. Before aggregation, all individual responses $<300 \mathrm{~ms}$ were discarded for a given task. Next, performance-based outliers were excluded. Specifically, participants were excluded from further analyses if their recall performance in any of the updating tasks fell $3 S D$ s above or below the respective grand mean of all participants $(n=9)$, if they completed a low number of trials on any of the updating tasks $(n=1)$, or if their RTs on the inhibition tasks $(n=5)$ or their age $(n=1)$ fell more than $3 S D$ s away from the mean.

\section{Updating tasks.}

Recall accuracy. As expected, recall accuracy was high for each updating task (letters: $M=.94, S D=.06$; digits: $M=.97, S D=.04$; words: $M=.93, S D=.06$ ). 
Updating RTs. Mean updating RTs for all tasks are provided in Figure 1. A $3 \times 2$ repeated measures ANOVA on updating RTs, with the factors task (letters, digits, words) and CTI (short, long) yielded a significant main effect of task, $F(2,242)=99.91, M S E=0.03$, $p<0.01, \eta_{\mathrm{p}}{ }^{2}=0.45$, a significant main effect of CTI, $F(1,121)=310.93, M S E=0.02, p<0.01$, $\eta_{\mathrm{p}}{ }^{2}=0.72$, as well as a slight but significant interaction, $F(2,242)=4.46, M S E=0.00, p=.013$, $\eta_{\mathrm{p}}{ }^{2}=0.04$. The main result of interest here was that, as expected, the effect of the CTI manipulation found by Ecker, Lewandowsky et al. (2014) was replicated across all three tasks.

Removal-efficiency scores. Three removal-efficiency scores per participant were calculated from the updating RTs of the three updating tasks. Removal-efficiency scores were calculated as regression residuals following Ecker, Lewandowsky et al. (2014). Table 1 displays descriptive statistics for the removal-efficiency scores; negative values on this score indicate greater removal efficiency and positive values indicating lower removal efficiency (with an expected mean of zero). Table 2 displays the inter-correlations of the removal-efficiency scores. All measures correlated moderately with each other, indicating that the tasks measured a common removal ability, but they did not correlate perfectly, indicating task-specific variance. Furthermore, Bartlett's test of sphericity was statistically significant, $\chi^{2}(3)=44.71, p<.001$. This indicated that a latent variable model could be specified.

Inhibition tasks. Table 1 displays descriptive statistics for the six inhibition tasks. Additionally, task inter-correlations are reported in Table 2 . The table shows weak, mostly nonsignificant correlations between the inhibition tasks.

Removal measurement model. The removal latent variable (REM) was defined by the removal-efficiency indices derived from the letter, digit, and word updating tasks. The REM latent variable had significant moderate positive standardized loadings from all three removal 
measures. The standardized loadings were $.43, .90$, and .49 for letters, digits, and words, respectively (all $p$ 's $<.001$ ). Model fit was necessarily perfect, as there were only three indicators specified to define a single-factor model. The substantial loadings indicated that the three tasks measured a common removal factor.

Inhibition measurement model. Initially, two inhibition measurement models were tested for each of the inhibition factors, RDI and RI. The RDI measurement model had nonsignificant $(p>.05)$ standardized loadings from its three observed variables, indicating that the three RDI tasks did not measure a common RDI factor. Similarly, the RI measurement model had non-significant $(p>.05)$ standardized loadings from its three observed variables, indicating that the three RI tasks did not measure a common RI factor. Consequently, a single-factor (general) inhibition measurement model was tested. This model also had non-significant ( $p>$ .05) standardized loadings from the six inhibition tasks, indicating that the inhibition tasks did not measure a common inhibition factor. Furthermore, Bartlett's test of sphericity was nonsignificant, $\chi^{2}(15)=17.87, p=.269$. In addition, the Kaiser-Meyer-Olkin (KMO) test of sampling adequacy indicated that the strength of the relationships among the inhibition tasks was low $(\mathrm{KMO}=.549)$ and below the commonly recommended value of .6 (Tabachnick \& Fidell, 2001). Taken together, these results suggest that conducting a latent variable analysis was not appropriate for the inhibition data. Consequently, an inhibition latent variable model was not specified.

Structural model. Given that a valid latent-variable model could not be specified for the inhibition data, a hybrid path analytic and structural equation model approach was employed. This approach allowed for the estimation of unique variance associated with the REM latent variable unaccounted for by the observed inhibition measures. Model solutions were estimated 
via maximum likelihood. Model-fit was evaluated with the following indices: the comparative fit index (CFI; Bentler, 1990), the Tucker-Lewis index (TLI; Tucker \& Lewis, 1973), the root mean square error of approximation (RMSEA; Browne \& Cudeck, 1993; Steiger \& Lind, 1980), and the standardized root mean square residual (SRMR; Bentler, 1995; Jöreskog \& Sörbom, 1986).

As can be seen in Figure 2, the hybrid model was defined by a REM latent factor defined by the three removal-efficiency measures. The REM latent variable was regressed upon the six inhibition indicators. In order to identify/scale the REM latent variable, the regression weight of one of the indicators (letter task measure) was fixed at 1. Due to the high loading of the digit task measure on the REM measurement model, the variance for this indicator was fixed to .001 in order to avoid a negative estimate. The REM latent variable was associated with a residual variance (i.e., rem_res), which represents the unique variance associated with the REM latent factor that is not accounted for by the inhibition measures. If most of the variance in the REM latent variable could be explained by the cumulative inhibition measures, the associated residual would be associated with a non-significant $p$-value. If there was unique variance associated with the REM latent variable, the associated residual would be associated with a significant $p$-value.

The model was found to be associated with a good level of fit ${ }^{2}, \chi^{2}(27)=32.51, p=.214$, $C F I=.906, T L I=.875, R M S E A=.041, S R M R=.076$. Additionally, the residual variance associated with the REM latent variable was statistically significant, rem_res $=.002, p=.024$. This indicated that there was unique variance associated with the $R E M$ latent variable that was not accounted for by the inhibition tasks, thus supporting the hypothesis that removal would be associated with unique true score variance. As can be seen in Table 3, none of the six beta

\footnotetext{
${ }^{2}$ The residual correlation between the antisaccade task (AS) and the local task (L) shown in Figure 2 improved model fit overall, but did not change any variable relations or the residual estimate of removal substantially.
} 
weights were associated with a statistically significant predictive capacity ( $p$-values ranged from .05 to .52$)$. Furthermore, the squared multiple correlation for the model was estimated as $R^{2}=.09$, and thus, only approximately $9 \%$ of the variance associated with the REM latent variable could be accounted for by the inhibition tasks. This was found not to be statistically significant based on 1,000 bootstrapped resamples, $p=.18$.

\section{Discussion}

The aim of this study was to investigate the relation between removal efficiency and inhibition. Previous research by Friedman and Miyake (2004) has identified an inhibitory process labelled resistance to distractor interference (RDI) that appears to share a similar function to the notion of removal (Ecker, Oberauer et al., 2014; Oberauer et al., 2012; Oberauer \& Lewandowsky, 2016) in that both processes involve preventing interference from distractors. The present study, therefore, aimed to investigate the extent to which removal can be characterized as an inhibitory executive process.

Given the multi-faceted nature of inhibition, two inhibitory factors were included in the present investigation: (1) RDI, a cognitive suppression process serving a similar function to removal; and (2) response inhibition (RI), a type of behavioural inhibition that acted as a control factor. It was hypothesised that if removal is a process of cognitive inhibition, removal ability would be related to RDI more so than RI. Alternatively, if removal can be characterized as a non-inhibitory executive function, as proposed by Ecker, Oberauer, and colleagues (Ecker, Lewandowsky et al., 2014; Ecker, Oberauer et al., 2014; Oberauer et al., 2012; Oberauer, Souza, Druey, \& Gade, 2013), then it was expected that there would be either no strong relation between removal and inhibition abilities, or that removal would relate to both factors of inhibition equally. 
A major limitation of the present study was the failure to observe an inhibition latent variable. The low correlations obtained between the inhibition tasks made it difficult to estimate (1) the extent to which removal and inhibition are related processes, and (2) the proportion of unique variance associated with each of these processes. Consequently, exploratory factor analytic methods were employed in order to assess the appropriateness of conducting a factor analysis given the low correlations between the inhibition tasks. Analyses indicated that latent variable analysis would not be appropriate for the inhibition data, thus a hybrid path analytic and structural equation modelling technique was employed, which attempted to explain variance in a latent removal variable from the inhibition measures. ${ }^{3}$

Failure to extract a latent inhibition factor is consistent with other independent studies that have also reported difficulty in obtaining an inhibition-specific factor using latent variable analysis (e.g., Huizinga, Dolan, \& van der Molen, 2006; van der Sluis, de Jong, \& van der Leij, 2007; see also Friedman \& Miyake, 2017; Hedge, Powell, \& Sumner, 2017). van der Sluis et al. suggested that this may be due to a number of reasons, including: (1) that inhibition may not be a reliable source of individual differences, at least in healthy functioning individuals; (2) that inhibition may be very difficult to measure reliably; and (3) that inhibition is highly correlated with other executive and non-executive processes. Friedman and Miyake $(2000,2004)$ reported low correlations between their inhibition task measures and large standard errors in their modelling, indicating that a large portion of variance for each inhibition task could not be accounted for by their respective latent inhibition factors. Specifically, the error variances for the inhibition measures reported by Friedman and Miyake (2004) ranged from .70 to .92 , indicating

${ }^{3}$ Of course, despite the fruitless attempt in the present study, future research should aim to reliably measure specific forms of inhibition at the latent-variable level, perhaps using a different set of tasks, and relate these latent inhibition measures to removal. 
that approximately 70 to $92 \%$ of the variance in the inhibition task measures could not be accounted for by a latent inhibition factor. The combination of low zero-order correlations and high error variance necessarily implies that the inhibition task measures used by Friedman and Miyake (2004), and other studies investigating inhibition more generally, make it difficult to reliably measure a latent inhibition factor. Studies investigating inhibition at the latent-variable level have often found that most of the variance that is common to inhibition tasks can be accounted for by another factor. For example, van der Sluis et al. (2007) and Friedman and Miyake (2017) found that most of the common variance from their inhibition tasks could be accounted for by naming and goal maintenance, respectively.

Regardless, the results from our modelling indicated that removal is associated with unique variance, providing tentative support that removal should not be considered an inhibitory process. This finding thus supports Ecker et al.'s (Ecker, Lewandowsky et al., 2014; Ecker, Oberauer et al., 2014) claim that active, item-wise removal ability is an independent executive process. In addition to this empirical evidence, there are also theoretical reasons that support the idea that removal should not be considered an inhibitory process. Specifically, Oberauer et al. (2013) presented a computational model suggesting that removal and inhibition operate at different levels. This model belongs to a class of models that, as discussed earlier, assumes that memory items in WM are bound to context, such as temporal order or list positions. Removing an item from WM essentially means "unbinding" an item from its context (e.g., unlearning the association between the number " 2 " and "position 1"). In contrast, inhibition is assumed to temporarily deactivate an item's representation more globally (e.g., suppressing the activation of the number " 2 " more generally). Thus, according to Oberauer et al.'s model, an item representation can be intentionally weakened through two mechanisms: (1) an item can be 
unlearned by gradually removing or unbinding the item from its context marker, resulting in impaired access to the item in WM; and (2) an item can be unlearned by inhibiting or suppressing its representation, resulting in a more general access deficit. Oberauer et al. therefore concluded that processes on bindings (i.e., facilitation, removal) are separate from processes on items (i.e., activation, inhibition).

Inhibition that operates on items should be evident in general access deficits that result from suppression. Such evidence comes from Healey et al. (2010) as well as Festini and ReuterLorenz (2013). Specifically, Healey et al. found that participants who successfully resolved interference between orthographically similar competing words (e.g., allergy and analogy) were subsequently slower to name the rejected word compared to participants who experienced no interference (e.g., liberty and analogy). Healey et al. argued that suppressing competing representations reduces the accessibility of those representations back to a baseline level, and that this reduced accessibility of distractor representations should be taken as a signature effect of inhibition. Indeed, Festini and Reuter-Lorenz (2013) also found that suppressed items are subsequently less accessible in a delayed recognition task.

Impaired access to an inhibited representation occurs most likely because inhibition reduces the activation of a memory representation, making the item temporarily less accessible during retrieval. The time course of this inhibitory process has been investigated in several electrophysiological studies using event-related potentials (ERPs). These can specify the time course of inhibition if there is a specific point in time (e.g., a cue onset) at which the requirement for inhibition is signalled. These studies have suggested that the electrophysiological correlates of inhibition can be seen as early as $200 \mathrm{~ms}$ post-stimulus onset (e.g., Falkenstein, Hohnsbein, \& Hoormann, 1999; Jodo \& Kayama, 1992). Additionally, ERP studies from the directed forgetting 
literature have suggested that, in general, it takes about 400-600 ms post-stimulus onset for inhibitory processes to peak (e.g., Norwicka, Jednoróg, Wypych, \& Marchewka, 2009; van Hooff \& Ford, 2011; van Hooff, Whitaker, \& Ford, 2009). Furthermore, they have suggested that inhibitory processes have a tendency to 'overshoot', such that the suppression does not only reduce an item's activity to baseline, but even below baseline (i.e., a temporary polarity reversal in the ERP approximately 600-1200 ms post-stimulus onset). This implies that the item becomes less accessible than a stimulus that was not recently activated (e.g., Norwicka, Jednoróg, Wypych, \& Marchewka, 2009; van Hooff \& Ford, 2011; van Hooff, Whitaker, \& Ford, 2009). At subsequent memory retrieval, this over-shoot can then manifest itself as a reversal of memoryrelated ERP effects. Specifically, ERPs in memory tasks show a well-studied pattern of old/new effects, where specific positive peaks in the ERP are enhanced by seeing previously studied items in comparison to unstudied items. Norwicka et al. (2009) and van Hooff et al. (2009) found a reversed old/new effect where studied but then inhibited items evoked less positive amplitudes than new words.

However, in searching for such a signature effect in behavioural data, Dagry et al. (2017) failed to find this item inhibition effect. In their study, Dagry et al. examined delayed recall and recognition of the distractor words of a complex span task, which were initially processed either at a fast or slow pace. Distractors processed at a slow pace were thought to provide more time to (1) attend to memory items and (2) delete distractors from the focus of WM, resulting in better WM performance. Thus, Dagry et al. expected a pace effect on the ease with which memory traces of these distractors were subsequently accessed: Distractors processed in the slow pace condition were expected to have undergone greater inhibition, and therefore were expected to be less accessible and more difficult to retrieve in delayed tests compared to distractors processed in 
the fast pace condition. Contrary to their expectations, Dagry et al. found that the pace at which distractors were processed did not affect subsequent recall and recognition of those distractors.

Dagry et al.’s (2017) findings go against both the inhibitory and the removal accounts of interference control. However, no strong conclusions can be made against either inhibition or removal because in their study, Dagry et al. confounded presentation and suppression/removal times — that is, Dagry et al.'s pace manipulation confounded the time available for distractor removal with the presentation time of distractors. Future research could overcome this by using Ecker et al.'s (Chang et al., 2017; Ecker, Lewandowsky et al., 2014; Ecker, Oberauer et al., 2014) removal task but with a more fine-grained manipulation of the CTI: If removal is an inhibitory process, then there may be a CTI that produces an RT decrement when an item is repeated or is similar to a previously presented item, and when combining this with an assessment of ERPs, there may be a polarity reversal in the associated ERP old/new effects. Such results could be taken as evidence for a signature inhibition effect, and would thus support the notion that removal is inhibitory. Thus, a focus for future studies should be to investigate the potential relationship between removal and inhibition using the modified updating paradigm introduced by Ecker et al. (Ecker, Lewandowsky et al., 2014; Ecker, Oberauer et al., 2014) to investigate the possible elimination of item repetition benefits at intermediate CTIs around 600 $1200 \mathrm{~ms}$.

Currently, however, the available evidence suggests that item repetition or similarity benefits are reduced — but do not disappear—with long CTIs (Ecker, Lewandowsky et al., 2014; Ecker, Oberauer et al., 2014). This falls more in line with an incomplete removal process rather than an inhibitory process. In further support, Oberauer and Vockenberg (2009) found that items in an updating task that were replaced but later reappeared in the same location actually 
continued to carry a small processing benefit, that is, showed slightly faster updating RTs, as opposed to the delay that might be expected if the outdated items were suppressed (cf. Dagry et al., 2017; Festini \& Reuter-Lorenz, 2017). This facilitation effect has been attributed to lingering residual bindings of the replaced item to the same location. Comparatively, repeated items that were replaced and reappeared in a different location suffered from slower updating RTs. This interference effect was likewise attributed to residual bindings of the replaced item to its old location.

Furthermore, as mentioned in the Introduction, it is acknowledged that a third inhibitory process - resistance to proactive interference (RPI) — has been identified by Friedman and Miyake (2004). While only two inhibition factors were selected for the present investigation based on previous research (i.e., Friedman \& Miyake, 2004), it can be argued further that removal shares a similar function to RPI, particularly when considering removal as a core updating process, in that both processes serve to discard previously relevant information from WM. Given the ambiguity surrounding RPI task measures - namely, whether they reflect interference protection in WM or LTM (Oberauer, 2009) - the link between the two cognitive inhibition factors proposed by Friedman and Miyake (2004), viz. RDI and RPI, should be reassessed by future research. Additionally, the potential relationship between removal and RPI may also be an area for future research.

To conclude, there remains a lack of consensus with respect to the types of tasks that should be used to measure the various types of inhibition (Friedman \& Miyake, 2004; Oberauer, 2009). We therefore argue, like others (e.g., Rey-Mermet, Gade, \& Oberauer, in press), that the term inhibition has perhaps been used too broadly to label different inhibitory processes that 
actually have little in common, and thus recommend that we call functions by their descriptive labels (e.g., removal) until replicable strong covariance is demonstrated. 


\section{Acknowledgements}

This research formed part of Kris Singh's PhD thesis at the University of Western Australia. It was facilitated by a University Postgraduate Award from the University of Western Australia to the first author. We thank Charles Hanich and Jia-Xin Tay for research assistance. The lab web address is http://www.cogsciwa.com. 


\section{References}

Anderson, M. C. (2001). Active forgetting: Evidence for functional inhibition as a source of memory failure. Journal of Aggression, Maltreatment, and Trauma, 4, 185-210.

Bentler, P. M. (1990). Comparative fit indexes in structural models. Psychological Bulletin, 107, 238-246.

Bentler, P. M. (1995). EQS structural equations program manual. Encino, CA: Multivariate Software.

Bissett, P. G., Nee, D. E., \& Jonides, J. (2009). Dissociating interference-control processes between memory and response. Journal of Experimental Psychology: Learning, Memory, and Cognition, 35, 1306-1316.

Briand, K. A. (1994). Selective attention to global and local structure of objects: Alternate measures of nontarget processing. Perception and Psychophysics, 55, 562-574.

Browne, M. W. \& Cudeck, R. (1993). Alternative ways of assessing model fit. In K. A. Bollen \& J. S. Long (Eds.), Testing structural equation models (pp. 136-162). Newbury Park, CA: Sage.

Bjork, R. A. (1989). Retrieval inhibition as an adaptive mechanism in human memory. In H. L. Roediger \& F. I. M. Craik (Eds.), Varieties of memory and consciousness: Essays in honor of Endel Tulving (pp. 309-330). Hillsdale, NJ: Erlbaum.

Chang, E. P., Ecker, U. K. H., \& Page, A. C. (2017). Impaired memory updating associated with impaired recall of negative words in dysphoric rumination - evidence for a removal deficit. Behaviour Research and Therapy, 93, 22-28. 
Conway, A. R. A., \& Engle, R. W. (1994). Working memory and retrieval: A resourcedependent inhibition model. Journal of Experimental Psychology: General, 123, 354373.

Dagry, I., Vergauwe, E., \& Barrouillet, P. (2017). Cleaning working memory: The fate of distractors. Journal of Memory and Language, 92, 327-342.

DeSchepper, B., \& Treisman, A. (1996). Visual memory for novel shapes: Implicit coding without attention. Journal of Experimental Psychology: Learning, Memory, and Cognition, 22, 27-47.

Ecker, U. K. H., Lewandowsky, S., \& Oberauer, K. (2014). Removal of information from working memory: A specific updating process. Journal of Memory and Language, 74, $77-90$.

Ecker, U. K. H., Lewandowsky, S., Oberauer, K., \& Chee, A. (2010). The components of working memory updating: An experimental decomposition and individual differences. Journal of Experimental Psychology: Learning, Memory, and Cognition, 36, 170-189.

Ecker, U. K. H., Oberauer, K., \& Lewandowsky, S. (2014). Working memory updating involves item-specific removal. Journal of Memory and Language, 74, 1-15.

Engle, R. W. (1996). Working memory and retrieval: An inhibition-resource approach. In J. T. E. Richardson, R. W. Engle, L. Hasher, R. H. Logie, E. R. Stoltzfus, \& R. T. Zacks, Working memory and human cognition. New York: Oxford University Press.

Engle, R. W., \& Kane, M. J. (2004). Executive attention, working memory capacity, and a twofactor theory of cognitive control. In B. Ross (Ed.), The psychology of learning and motivation (pp. 145-199). New York: Academic Press. 
Engle, R. W., Kane, M. J., \& Tuholski, S. W. (1999). Individual differences in working memory capacity and what they tell us about controlled attention, general fluid intelligence and functions of the prefrontal cortex. In A. Miyake \& P. Shah (Eds.), Models of working memory: mechanisms of active maintenance and executive control (pp. 102-134). New York: Cambridge University Press.

Eriksen, B.A., \& Eriksen, C.W. (1974). Effects of noise upon the identification of a target latter in a nonsearch task. Perception \& Psychophysics, 16, 143-149.

Falkenstein, M., Hoormann, J., \& Hohnsbein, J. (1999). ERP components in go/nogo tasks and their relation to inhibition. Acta Psychologica, 101, 267-291.

Fan, X. (2003). Two approaches for correcting correlation attenuation caused by measurement error: Implications for research practice. Educational and Psychological Measurement, 63, 915-930

Farrell, S., \& Lewandowsky, S. (2002). An endogenous distributed model of ordering in serial recall. Psychonomic Bulletin \& Review, 9, 59-79.

Fawcett, J. M., \& Taylor, T. L. (2008). Forgetting is effortful: Evidence from reaction time probes in an item-method directed forgetting task. Memory and Cognition, 36, 11681181.

Fawcett, J. M., \& Taylor, T. L. (2010). Directed forgetting shares mechanisms with attentional withdrawal but not stop-signal inhibition. Memory and Cognition, 38, 797-808.

Fawcett, J. M., \& Taylor, T. L. (2012). The control of working memory resources in intentional forgetting: Evidence from incidental probe word recognition. Acta Psychologica, 139, 84-90. 
Festini, S. B. \& Reuter-Lorenz, P. A. (2013). The short- and long-term consequences of directed forgetting in a working memory task. Memory, 21, 763-777.

Festini, S. B. \& Reuter-Lorenz, P. A. (2017). Rehearsal of to-be-remembered items is unnecessary to perform directed forgetting within working memory: Support for an active control mechanism. Journal of Experimental Psychology: Learning, Memory, and Cognition, 43, 94-108.

Friedman, N., \& Miyake, A. (2004). The relations among inhibition and interference control functions: A latent-variable analysis. Journal of Experimental Psychology: General, 133, 101-135.

Friedman, N., \& Miyake, A. (2017). Unity and diversity of executive functions: Individual differences as a window on cognitive structure. Cortex, 86, 186-204.

Hallett, P.E. (1978). Primary and secondary saccades to goals defined by instructions. Vision Research, 18, 1279-1296.

Harnishfeger, K. K. (1995). The development of cognitive inhibition: Theories, definitions, and research evidence. In F. N. Dempster \& C. J. Brainerd (Eds.), Interference and inhibition in cognition (pp. 175-204). New York, NY: Academic Press.

Hasher, L., Lustig, C., \& Zacks, R. T. (2007). Inhibitory mechanisms and the control of attention. In A. Conway, C. Jarrold, M. Kane, A. Miyake, \& J. Towse (Eds.), Variation in working memory (pp. 227-249). New York: Oxford University Press.

Hasher, L., Zacks, R. T., \& May, C. P. (1999). Inhibitory control, circadian arousal, and age. In D. Gopher \& A. Koriat (Eds.), Attention and performance: XVII. Cognitive regulation of performance: Interaction of theory and application (pp. 653-675). Cambridge, MA: MIT Press. 
Healey, M. K., Campbell, K. L., Hasher, L., \& Ossher, L. (2010). Direct evidence for the role of inhibition in resoling interference in memory. Psychological Science, 21, 1464-1470.

Hedge, C., Powell, G., \& Sumner, P. (2017). The reliability paradox: Why robust cognitive tasks do not produce reliable individual differences. Behavior Research Methods, 1-21.

Huizinga, M., Dolan, C. V., \& van der Molen, M. W. (2006). Age-related change in executive function: Developmental trends and a latent variables analysis. Neuropsychologia, 44, 2017-2036.

Jodo, E., \& Kayama, Y. (1992). Relation of a negative ERP component to response inhibition in a go/nogo task. Electroencephalography and Clinical Neurophysiology, 82, 477-482.

Jöreskog, K. G., \& Sörbom, D. (1986). LISREL VI: Analysis of linear structural relationships by maximum likelihood and least squares methods. Mooresville, IN: Scientific Software.

Kane, M. J. \& Engle, R. W. (2000). Working-memory capacity, proactive interference, and divided attention: Limits on long-term memory retrieval. Journal of Experimental Psychology: Learning, Memory, and Cognition, 26, 336-358.

Kane, M. J., \& Engle, R. W. (2003). Working-memory capacity and the control of attention: The contributions of goal neglect, response competition, and task set to Stroop interference. Journal of Experimental Psychology: General, 132, 47-70.

Kane, M. J., Hasher, L., Stoltzfus, E. R., Zacks, R. T., \& Connelly, S. L. (1994). Inhibitory attentional mechanisms and aging. Psychology and Aging, 9, 103-112.

Kessler, Y., \& Meiran, N. (2008). Two dissociable updating processes in working memory. Journal of Experimental Psychology: Learning, Memory, and Cognition, 34, 1339-1348. 
Lendínez, C., Pelegrina, S., \& Lechuga, T. (2011). The distance effect in numerical memoryupdating tasks. Memory \& Cognition, 39, 675-685.

Logan, G. D. (1994). On the ability to inhibit thought and action: A user's guide to the stop signal paradigm. In D. Dagenbach \& T. H. Carr (Eds.), Inhibitory processes in attention, memory, and language (pp. 189-239). San Diego, CA: Academic Press.

MacLeod, C. M. (2007). The concept of inhibition. In D. S. Gorfein \& C. M. MacLeod (Eds.), Inhibition in cognition (pp. 3-23). Washington, D.C.: American Psychological Association.

Miyake, A., \& Shah, P. (1999). Models of working memory: Mechanisms of active maintenance and executive control. Cambridge University Press.

Navon, D. (1977). Forest before trees: the precedence of global features in visual perception. Cognitive Psychology, 9, 353-383.

Nigg, J. T. (2000). On inhibition/disinhibition in developmental psychopathology: Views from cognitive and personality psychology and a working inhibition taxonomy. Psychological Bulletin, 126, 220-246.

Norwicka, A., Jednoróg, K., Wypych, M., \& Marchewka, A. (2009). Reversed old/new effect for intentionally forgotten words: An ERP study of directed forgetting. International Journal of Psychophysiology, 71, 97-102.

Oberauer, K. (2009). Design for a working memory. Psychology of Learning and Motivation, 51, 45-100.

Oberauer, K. \& Lewandowsky, S. (2016). Control of information in working memory: Encoding and removal of distractors in the complex-span paradigm. Cognition, 156, 106-128. 
Oberauer, K., Lewandowsky, S., Farrell, S., Jarrold, C., \& Greaves, M. (2012). Modeling working memory: An interference model of complex span. Psychonomic Bulletin \& Review, 19, 779-819.

Oberauer, K., Souza, A. S., Druey, M. D., \& Gade, M. (2013). Analogous mechanisms of selection and updating in declarative and procedural working memory: Experiments and a computational model. Cognitive Psychology, 66, 157-211.

Oberauer, K., \& Vockenberg, K. (2009). Updating of working memory: Lingering bindings. The Quarterly Journal of Experimental Psychology, 62, 967-987.

Rey-Mermet, A., Gade, M., \& Oberauer, K. (in press). Should we stop thinking about inhibition? Searching for individual and age differences in inhibition ability. Journal of Experimental Psychology: Learning, Memory, and Cognition.

Rosen, V. M., \& Engle, R. W. (1998). Working memory capacity and suppression. Journal of Memory and Language, 39, 418-436.

Simon, J. R. \& Ruddell, A. P. (1967). Auditory S-R compatibility: The effect of an irrelevant cue on information processing. Journal of Applied Psychology, 51, 300-304.

Singh, K. A., Ecker, U. K. H., \& Gignac, G. E. (2017). Removal of information from working memory relates to working memory capacity and fluid intelligence. Unpublished manuscript, School of Psychological Sciences, University of Western Australia, Perth,

Steiger, J. H. \& Lind, J. C. (1980, June). Statistically based tests for the number of common factors. Paper presented at the Psychometric Society Annual Meeting, Iowa City, IA.

Stroop, J.R. (1935). Studies of interference in serial verbal reactions. Journal of Experimental Psychology, 18, 643-662. 
Sumner, P. (2008). Mask-induced priming and the negative compatibility effect. Experimental Psychology, 55, 133-141.

Tabachnick, B. G., \& Fidell, L. S. (2001). Using multivariate statistics $\left(4^{\text {th }}\right.$ ed.) New York, NY: Harper \& Row.

Tolan, G. A., \& Tehan, G. (1999). Determinants of short-term forgetting: decay, retroactive interference, or proactive interference? International Journal of Psychology, 34, 285-292.

Tucker, L. R., \& Lewis, C. (1973). A reliability coefficient for maximum likelihood factor analysis. Psychometrika, 38, 1-10.

Unsworth, N. (2010). Interference control, working memory capacity, and cognitive abilities: a latent variable analysis. Intelligence, 38, 255-267.

van der Maas, H. L. J., Dolan, C. V., Grasman, R. P. P. P., Wicherts, J. M., Huizenga, H. M., \& Raijmakers, M. E. J. (2006). A dynamic model of general intelligence: The positive manifold of intelligence by mutualism. Psychological Review, 113, 842-861.

van der Sluis, S., de Jong, P. F., van der Leij, A. (2007). Executive functioning in children, and its relations with reasoning, reading, and arithmetic. Intelligence, 35, 427-449.

van Hooff, J. C., \& Ford, R. M. (2011). Remember to forget: ERP evidence for inhibition in an item-method directed forgetting paradigm. Brain Research, 1392, 80-92.

van Hooff, J. C., \& Whitaker, T. A., \& Ford, R. M. (2009). Directed forgetting in direct and indirect tests of memory: Seeking evidence of retrieval inhibition using electrophysiological measures. Brain and Cognition, 71, 153-164.

Zacks, R. T., Radvansky, G., \& Hasher, L. (1996). Studies of directed forgetting in older adults. Journal of Experimental Psychology: Learning, Memory, and Cognition, 22, 143-156. 
Table 1.

Descriptive Statistics and Reliability Estimates for the Removal and Inhibition Tasks.

\begin{tabular}{|c|c|c|c|c|c|c|c|}
\hline \multirow[t]{2}{*}{ Task } & \multirow[t]{2}{*}{ Mean } & \multirow[t]{2}{*}{$S D$} & \multirow[t]{2}{*}{ Range } & \multirow[t]{2}{*}{ Skewness } & \multirow[t]{2}{*}{ Kurtosis } & \multicolumn{2}{|c|}{ Reliability } \\
\hline & & & & & & Accuracy & $\mathrm{RT}$ \\
\hline RemL & 0.00 & 0.12 & $-0.24-0.43$ & 0.79 & 1.34 & & \\
\hline RemD & 0.00 & 0.12 & $-0.35-0.46$ & 1.12 & 2.90 & & \\
\hline RemW & 0.00 & 0.11 & $-0.36-0.31$ & 0.15 & 0.81 & & \\
\hline $\mathrm{AF}$ & 21.44 & 23.55 & $-83.73-76.07$ & -1.02 & 3.56 & .09 & .63 \\
\hline AS & 122.64 & 136.35 & $-271.84-645.10$ & 0.50 & 1.61 & .92 & .90 \\
\hline $\mathrm{L}$ & 23.12 & 35.26 & $-53.60-136.54$ & 0.89 & 1.28 & .13 & .52 \\
\hline $\mathrm{NC}$ & -12.93 & 24.21 & $-71.33-120.91$ & 1.28 & 7.02 & .48 & -.01 \\
\hline NS & 64.57 & 26.62 & $-7.13-143.36$ & 0.56 & 0.94 & .72 & .35 \\
\hline S & 24.12 & 33.30 & $-93.28-131.23$ & 0.17 & 2.24 & .63 & .65 \\
\hline
\end{tabular}

Note. Removal tasks: RemL = letter updating; RemD = digit updating; RemW = word updating. Inhibition tasks: $\mathrm{AF}=$ arrow flanker; $\mathrm{AS}=$ antisaccade task $\mathrm{L}=$ local task (from global-local task); NC = negative compatibility task; NS = number Stroop task; $\mathrm{S}=$ Simon task. The $M$ 's for the letter, digit, and word updating tasks were based on regression-residual scores, calculated as the individual residuals obtained from a simple linear regression model predicting the short-CTI RTs from the long-CTI RTs. The $M$ 's and SD's for the inhibition tasks are in milliseconds. Reliability estimates for the inhibition tasks are split-half Spearman-Brown corrected reliabilities. $N=122$. 
Table 2.

Task Inter-correlations Between Removal Efficiency Scores and Inhibition tasks.

\begin{tabular}{|c|c|c|c|c|c|c|c|c|c|}
\hline Task & 1 & 2 & 3 & 4 & 5 & 6 & 7 & 8 & 9 \\
\hline 1. RemL & - & & & & & & & & \\
\hline 2. RemD & $.37 * *$ & - & & & & & & & \\
\hline 3. RemW & $.21 *$ & $.44 * *$ & - & & & & & & \\
\hline 4. $\mathrm{AF}$ & -.03 & .03 & .09 & - & & & & & \\
\hline 5. AS & $-.20 *$ & -.12 & -.05 & .12 & - & & & & \\
\hline 6. L & $-.19 *$ & -.12 & .06 & .09 & $.25^{* *}$ & - & & & \\
\hline 7. NC & -.08 & .17 & -.09 & .01 & .15 & .12 & - & & \\
\hline 8. NS & .11 & .07 & .04 & -.03 & .06 & -.00 & -.01 & - & \\
\hline 9. $\mathrm{S}$ & $.22 *$ & .09 & -.00 & -.07 & -.04 & -.12 & -.10 & .04 & - \\
\hline
\end{tabular}

Note. Removal tasks: RemL = letter updating; RemD = digit updating; RemW = word updating. Inhibition tasks: $\mathrm{AF}=$ arrow flanker task; $\mathrm{AS}=$ antisaccade task; $\mathrm{L}=$ local task (from globallocal task); NC = negative compatibility task; NS = number Stroop task; $\mathrm{S}=$ Simon task. $N=122 . * * p<.001 ; * p<.05$. 
Table 3 .

Beta weights for the hybrid path analytic and structural equation model from Figure 3.

\begin{tabular}{lcccc}
\hline Variable & Unstandardised & Standardised & CI & $p$ \\
\hline RemL & 1.00 & .41 & $.21-.56$ & .003 \\
RemD & 2.44 & .97 & $.95-.98$ & $<.001$ \\
RemW & 1.05 & .45 & $.29-.59$ & .001 \\
AF & .00 & .06 & $-.11-.23$ & .52 \\
AS & -.00 & -.14 & $-.31-.03$ & .16 \\
L & -.00 & -.11 & $-.26-.02$ & .24 \\
NC & -.00 & -.19 & $-.35--.02$ & .05 \\
NS & .00 & .08 & $-.17-.26$ & .36 \\
S & .00 & .06 & $-.16-.25$ & .51 \\
& & & & \\
\hline
\end{tabular}

Note. Removal tasks: RemL = letter updating; RemD = digit updating; RemW = word updating. Inhibition tasks: $\mathrm{AF}=$ arrow flanker task; $\mathrm{AS}=$ antisaccade task; $\mathrm{L}=$ local task (from global-local task); NC = negative compatibility task; NS = number Stroop task; $\mathrm{S}=$ Simon task. $\mathrm{CI}=95 \%$ confidence interval. $N=122$. 
Figure 1.

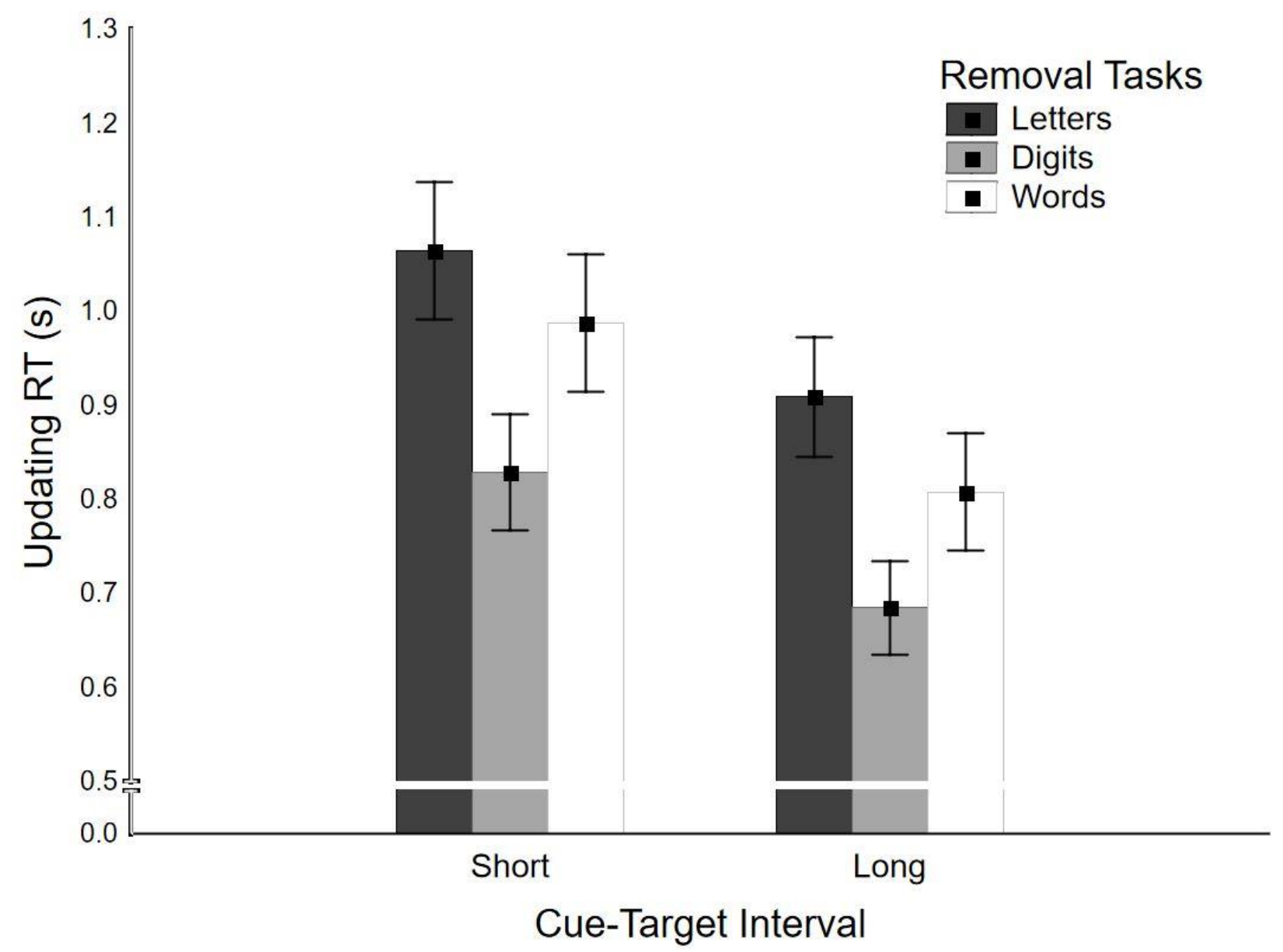

Figure 1. Updating response times for the three removal tasks by CTI condition for Study 5.

Short CTI $=200 \mathrm{~ms}$; long CTI $=1,500 \mathrm{~ms}$. Vertical bars denote within-subject standard errors of the mean. 
Figure 2.

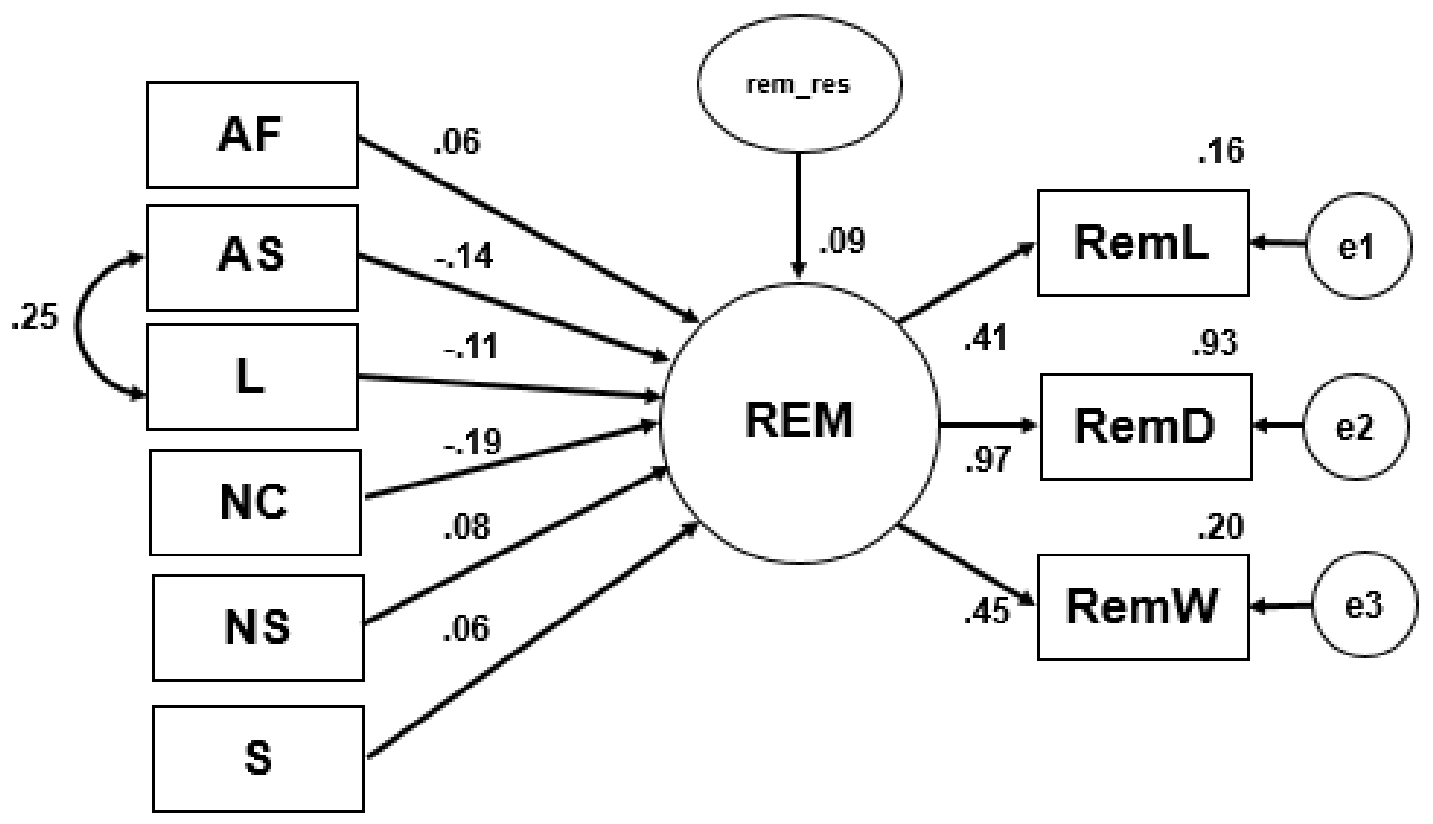

Figure 2. A hybrid path analytic latent variable model regressing removal efficiency (REM) onto six indicators of inhibition (for acronym spellings, consult notes in Tables $1-3$ ). e1-e3 = error variables. 